\title{
Challenge and relief: A Foucauldian analysis of retirement from professional association football in the United Kingdom \\ Luke Jones \\ Jim Denison
}

\begin{abstract}
The aim of this study was to consider the retirement experiences of British male professional Association footballers by utilising Foucault’s (1991) analysis of discipline discussed in Discipline and Punish: the Birth of the Prison. Specifically, we drew upon Foucault to consider how, through the various techniques and instruments of discipline, the professional football context produces 'docile footballing bodies' and how this might influence a player’s experiences in retirement. We gathered our empirical material using a Foucauldian-informed interview framework (Avner et al., 2013) with 25 former professional male football players between the ages of 21-34. Our analysis suggested that retirement from football was both a challenge and a relief for our participants, and that their extended period of time within football's strong disciplinary apparatus significantly influenced how they experienced their retirement.
\end{abstract}

Keywords: sport retirement; football; Foucault, discipline; docility 
According to a number of sports scholars working across a range of disciplines, the process of retirement from sport can be a challenging period in the lives of elite sportspersons (e.g., Blinde and Stratta, 1992; Fuller, 2014; Sinclair and Orlick, 1993; Wylleman et al., 2004). At the same time, other sports scholars have suggested that for many athletes retiring from elite sport can be a relief and represent a new beginning to life rather than an end to life (e.g., Coakley, 1983; Stier, 2007). In an effort to explore both the challenge and relief of sport retirement, we ask in this paper, how might the everyday and 'normal' practices of 'doing sport' and 'being an athlete' effect athletes' retirement experiences? More specifically, as Foucauldians what interests us is how athletes' exposure to a range of disciplinary practices over the course of their career might influence how they make sense of their retirement from sport. And one sport where we believe an examination of how the exposure to discipline on a regular basis could influence an athlete's experiences in retirement is professional football. The relationship between discipline and football has been highlighted by existing sociocultural research (Giulianotti, 1999). For example, according to Christensen (2009) and Cushion and Jones (2006), a working footballer's body is identified, then exposed to a disciplinary lifestyle that targets and transforms his/her body to produce exceptional and consistent athletic performances. Despite this acknowledgement that football is a highly disciplinary space, according to Curran (2015) and Roderick (2006), for the working footballer, the long term effects of living this lifestyle are largely unknown. Therefore, 
in order to understand how the disciplinary nature of football, and the way in which players' bodies are transformed and made useful in specific ways, might impact players’ retirement experiences, we interviewed 25 British retired professional male players about their life in and out of football. In what follows, we discuss in detail our Foucauldian theoretical framework for conducting this study, after which we present our results and what we believe their implications are for future sport retirement research.

\section{Thinking about retirement from football with Foucault}

It is our intention to remind the reader that as Foucauldian scholars we do not promote our use of the term 'retirement' here as an absolute or finite category.

Retirement from sport is, after all, an immensely complex phenomenon that individuals experience in a multitude of ways. However, in this particular instance we use the term 'retirement' to convey that our participants were once deeply invested and connected to an operational realm, and now find themselves removed from that familiar space.

Roderick (2012) reported that 600-700 male football players are released from the professional game each season in the United Kingdom, indicating that around the globe thousands of men experience retirement from football annually. Football is a difficult and risky landscape into which a large number of young men commit themselves in an attempt to build careers that are socially canonised by their immediate culture (McGillivray et al., 2005). In 2013 the Professional Football Association (PFA) 
in Britain revealed that currently there are over 150 former male professionals in the British prison system. Men's professional football also has a history of depression and suicide among current and former players (Curran, 2015; Turner et al., 2000). For example, this past year (2014), Clarke Carlisle, a former English Premier League footballer and the former head of the PFA attempted to take his own life. While Gearing (1997) reported that the length of a footballer's career is on average eight and a half years, most players' retirement from professional football happens during their adolescent years (Brown and Potrac, 2009), a period when athletic identity associated with a football role is most ingrained (Mitchell et al., 2014). As a result of these various issues, and the effects associated with a life after professional football, along with Roderick (2006), we believe that in order to better understand what retirement for footballer players means, greater consideration needs to be given to the types of practices and relationships athletes are exposed to during their careers and what the implications of these practices and relationships are for their retirement. More specifically, we believe that by thinking about retirement from football with Foucault (1991), we might better understand how disciplinary power operates in football and what consequences this can have for players when they retire.

\section{A disciplinary analysis of working football}

Foucault (1991: 137) considered discipline to be a technique of dominance that worked to make the body both an object and target of power. It is a technique 
specifically designed to achieve control and efficiency and “make useful individuals” by classifying and normalising them. It is no surprise therefore that Foucault's analysis of discipline has been utilised to problematise elite sport, an area where the exercise discipline is widely celebrated (e,g., Heikkala, 1993; Shogan, 1999).

In addition, Foucault’s (1991) disciplinary analysis has proven to be an appropriate theoretical framework to identify and problematise many taken-for-granted disciplinary coaching practices across elite sport (e.g., Barker-Ruchti and Tinning, 2010; Denison, 2007; Denison and Avner, 2011; Gearity and Mills, 2012). For example, Shogan (1999) utilised Foucault's analysis of discipline to reveal the power laden and normalising nature of every day coaching practices. In doing so she highlighted a number of limiting and unintended consequences that these coaching practices can have for an athlete including under performance (Denison, 2007) and disordered eating (Johns and Johns, 2000). For our purposes here, we intend to use Foucault's analytical techniques and instruments of discipline (outlined below) to consider how players’ exposure to, and removal from, the localised disciplinary space of football might impact their retirement experiences.

Key to what we will discuss in this paper is Foucault's (1991) observation that through the exercise of disciplinary power, individual bodies become 'docile'. In this way, Foucault was interested in discipline not only because of its productive effects, but also because of the way in which it objectified and normalised individuals. Foucault 
saw discipline’s techniques and instruments as the primary way in which power, through subtle coercion, made individuals docile. Foucault suggested that within institutional spaces (such as a football club), disciplinary power operated through the fabrication of individuals into an organised social order: "discipline produces subjected and practised bodies, 'docile’ bodies'... that may be subjected, used, transformed and improved” (136). During his career a footballer’s body, according to Roderick (2006) and Giulinaotti (1999), is manipulated and given a 'function’ in order to be productive (read a useful commodity). In this way, Foucault’s (1991: 136) description of a docile body, where a body “is manipulated, shaped, trained...obeys, responds, becomes skilful and increases its forces” could be said to define what it means to be a footballer. Therefore, in what follows we utilise Foucault's (1991) analysis of docility in an effort to establish how discipline operates at the localised sight of the football club to make players docile and subsequently what consequences this might have for a player once he exits the game.

To explain the precise details of how bodies are made docile through disciplinary power, Foucault (1991) outlined four specific techniques or 'disciplines’. More specifically, he illustrated how these techniques "made possible the meticulous control of the operations of the body” (137). Foucault's first technique, the art of distributions, involved how bodies were managed and used in spaces. Foucault's second technique, what he called the control of activity, explained how the body was shaped by 
time, in these spaces. Foucault's third technique involved the way specific bodily practices were categorised and grouped to ensure the "continual growth of control over the body” (Markula and Pringle, 2006: 78). This progressive arrangement of activity he described as the organisation of geneses. In presenting this particular technique of discipline, Foucault revealed that in order to continue to exercise disciplinary power over an individual, his/her body must repeatedly be exposed to activities that allow for a “linear progression” (Markula and Pringle, 2006: 78) and the development of the education of the body. Foucault's fourth technique, therefore, the composition of forces, involved the way in which bodies were brought together to function as a machine. Accordingly for Foucault, discipline was not simply about correction and instruction; it was also focused upon the elements of efficiency, productivity and usefulness (Denison et al., 2015).

Importantly for Foucault (1991), these four techniques that we outlined above did not operate in a disjointed or fragmented manner; rather they worked together to exert their influence over the body through three specific instruments: hierarchical observation, normalizing judgment and in the examination. Underpinning Foucault's (1991) analysis of disciplinary power and the making of docile bodies was his understanding of panopticism, a concept he based on Jeremy Bentham’s architectural figure of the panopticon. From the total force of this analysis, Foucault was able to show how in large social institutions where disciplinary regimes are prevalent (such as 
factories, the military, schools and hospitals), people can easily be transformed into cogs in a system where interaction, learning and personal growth are subservient to the large scale production of all: “an infinitesimal power over the active body...its movements, gestures, attitudes, rapidity” (137). And working football is one particular modern institution where these disciplinary regimes have been shown to be clearly present (Giulianotti, 1999). For this reason, we intend to use Foucault’s (1991) analysis of the techniques and instruments of discipline to examine the long-term implications of these regimes upon working players. In what follows we outline our methodology and explain how we examined the retirement experiences of working British footballers in order to answer the question: what about the docile body in retirement?

\section{Method}

We subscribe to the tenets of post-structuralism. Post-structuralism suggests that power is relational (Markula and Silk, 2011), that knowledge is contextual, and that 'reality' and 'truth’ are both multiple and subjective (Avner et al., 2014). Since the emergence of post-structurally informed sports sociology (much of which adopts the work of Foucault) a more critical awareness of taken for granted and potentially dangerous practices that operate within sport has emerged (e.g., Markula and Pringle, 2006). Given Foucault’s post-structural position that power, knowledge, and truth are “produced rather than found” (Avner et al., 2014: 43), it is clear that there was no concrete 'truth' to discover about retirement from football. Rather, this post-structuralist 
study aimed to subjectively analyse the dominant relations of power, coaching practices and knowledge found in the sporting context of working football in order to trace how the disciplinary context of football might have contributed to a player's retirement experiences.

This study utilised semi-structured interviews (Patton, 2002) to collect data from 25 British male retired football players between the ages of 21-34 (average age 29). Each player had to have played his last game at an elite level (between the Premier League and Conference (now National League) level) within three years of being interviewed. The average length of our participants’ playing careers was just over three years. Now in their post-retirement lives, one of our participants works as a financial advisor in the property sector while the rest of our participants currently occupy four main roles: working within elite football as coaches /development officers/personal trainers, working as teachers or assistants in education (predominantly Physical Education), working as manual/technical labourers, or working to pursue a further qualification in higher education.

Our interview guide was specifically devised after lengthy engagement with the analytical tools Foucault (1991) discussed in Discipline and Punish and that we outlined in the previous section. Using this interview guide, it was our aim to expose the detailed workings and effects of the various aspects of discipline a player experienced during his career in order to make the connection between these experiences and those of 
retirement. As a former youth international and National League level player, the first author was able to sustain a lively and frank interview discussion with each participant. We believe this factor was important for this particular study as the football environment has its own particular vocabulary (Roderick, 2006). The first author did his best to create an environment in which the participants' interpretive capabilities were "activated, stimulated, and cultivated" (Holstein and Gubrium, 1995: 17) so that they could relax and use their own mutually understood terminology to reveal their experiences of retirement. Our questions intended to: a) expose the various spatial and temporal components of football, b) discuss the hierarchical relationships common to a football space, c) highlight the surveillance a player experienced during his working football career, and d) discuss the implications of living within this disciplinary arrangement when faced with retirement. Our empirical data was scrutinized using Foucault's analytical tools in order to expose the detailed workings and effects of discipline in the football context. Using Foucault's disciplinary framework has allowed us to frame retirement as the removal of an individual from an overtly disciplinary context. In the discussion and analysis sections that follow, we have considered the relationship between exposure to discipline in a football context and becoming a 'docile footballing body', and the subsequent retirement experiences of each player we interviewed. In total this process allowed us to answer the following question: how, if at 
all, does experiencing life as a 'docile football body' influence the various conflicting emotional responses to retirement?

\section{A disciplinary analysis of the experiences of retirement from working football}

As mentioned above, the predominant finding from our interviews was that retirement is simultaneously both a challenge and a relief for the majority of retiring players. A quote from Paul, a former member of a Premier League first team squad, illustrates these paradoxical feelings:

Coming out of the full-time game is a lot harder than people imagine. Many retiring players really struggle just trying to find a job. They are in the same boat as people just coming out of school. But, at the same time it is a real release, you know? It's like the pressure has just evaporated.

Paul's quote reveals that in retirement, a footballing identity can no longer be sustained and that an alternative understanding of one's role in life becomes a necessity. We have found that the responsibilities that accompany retirement are both a shock and a relief to a player's system. The repeated reference to a shock to the system also highlights just how insulated from many of life's everyday concerns players become during their careers.

In order to understand the experiences of retiring players, we suggest that it makes sense to consider retirement from football as governed by exposure to, and removal from, a space of localised disciplinary power (Foucault, 1991). In this regard, 
we discuss how the disciplinary elements common to football are sustaining for a working player, and demonstrate why the removal of this discipline can be so challenging. We also discuss how the disciplinary nature of football has multiple punitive effects for the working player. As a result, we argue that retirement, and moving away from these punitive effects, can simultaneously be a distinct relief for players. 


\section{Challenging times}

Despite the uncertainty of a football career (Roderick, 2006), football as a vocation still provided our participants with a clear identity, allowing them to exist within a constant space where (through the regular absorption of discipline) their lives had an established focus and direction. However, as a result of career-ending injury or de-selection, retirement from football can be painful and abrupt. Access to the familiar and sustaining disciplinary lifestyle is revoked, and this presents a significant challenge for the retiring player. In the discussion that follows we establish how the regular absorption of discipline occurs in a football context and go on to explain why removal from this protective 'bubble like’ space (McGillivray et al., 2005) is such a challenge. Specifically, we examine the physical and relational arrangement of the social spaces a footballer occupies. And, we consider how the design and regulation of these spaces exposed him to a heavily orchestrated and monitored disciplinary lifestyle. We begin by considering how Foucault's disciplinary techniques are useful for explaining the working lifestyle of a footballer and the production of a docile footballer. We then move on to consider how Foucault's instruments of surveillance, or his 'Means of correct training', can be used to more clearly understand the discipline experienced during a career. 
The initial experience of retirement can clearly be a painful experience. When a club director informed him of his release from his club, Andrew recalled that he, felt terrible. I felt let down and massively disappointed. I was angry. As I was driving home from the game I was devastated, I cried all the way home...I remember driving to a local park and sitting on a bench for hours crying my eyes out, ignoring phone calls. I was just thinking, 'What the fuck am I doing?' and that football was shit and everything was going to pot. I just needed something else.

In another example, one of the longest serving players we interviewed, Brian, spoke about the anxiety he felt over his future when he came to the end of his eight year career:

I have no qualifications from the game, just memories...I remember that I went to the cash point and I couldn’t even take any money out. I remember wandering around on the beach thinking, 'How am I going to get money? I can’t even pay the mortgage'. Then the realisation hit me hard. Up until then I had been wrapped in cotton wool.

The 'cotton wool' that Brian spoke of is an excellent description of the disciplinary lifestyle he was accustomed to in working football. Using Foucault's analytical tool 'the art of distributions', it is possible to more clearly explain how disciplinary power focused upon a footballer's body during his career. As a footballer, a player is required 
to arrive at the same designated space at the same time every day. He is expected to train within the 'enclosure' of his club’s training facilities (pitches/gymnasium etc), in accordance with his 'rank' (first team squad member). As Liam mentioned, It was the same routine every day. $8.30 \mathrm{am}$. Turn up at the training ground, out for a warm up, then some ball work. At 9.30 am on the dot, a short, sharp first session. Usually keep-ball, then we would break up into groups. Sometimes the whole squad joined in but mostly the lads playing on the Saturday would head off and work with the coaches and do shape. If you weren't involved, you either shadowed the first team or went off and did shooting, extra running, hit the gym, anything to try and get back in the fold.

Liam identified that during a training session, he was required to follow the instructions of those of superior rank, specifically the coaching staff. Furthermore, if he was not involved in the first team Liam was expected to engage in extra work away from training in a self-conscious attempt to improve his 'function' for his team.

Like all retired players, Liam and Brian no longer belong to football. Their positions or 'rank' within the socially desirable 'club' they worked so hard for has been revoked. As footballers, their focus and role had been made abundantly clear to them by the disciplinary tone of their relationships and activities. As Brian said, 
When I played football I always knew what was expected of me. I knew what to focus upon on a daily basis. It was simple. Train hard all week. Do what the coaches said. Stay in the team. Keep your head down and stay out of trouble. Foucault's notion of the 'control of activity’ can be helpful here to understand Brian’s experiences. For example, when acting as a footballer, Brian’s focus was explicitly enacted through the intentional design of the activities he was expected to complete on a daily basis, as well as the strict tone in which these activities were progressively controlled via 'timetables' in order to facilitate his improvement. Almost all of our participants reported that when the focus that daily discipline allowed was removed, when it no longer became available as a result of retirement, a significant struggle ensued. Michael explained that,

I had no direction. I didn’t know what to do with myself - my days had no pattern to them and that really got me down. I would lie in bed in the morning and just not want to get up because I knew that the day had no purpose to it. I didn't want to be exposed to everyday things, and then I spiralled further and further into a dark place.

As a result of the daily training routines and disciplinary lifestyle reported by the majority of our participants it is clear that they experienced being disciplined into ‘docile footballing bodies’ by the practices demanded by the role of a working footballer. We would argue that Michael's reaction to retirement occurred because 
without the control of his activities, the micro-management of his development, what Foucault called the 'organisation of geneses', or the efficient and economic use of his footballing body in relation to a football, what Foucault called 'body object articulation', he no longer felt protected by the structured disciplinary lifestyle he was accustomed to. One could argue that as a result of the removal of what Brian called the 'cotton wool' of football, Michael experienced his downward spiral.

Clearly, a player's removal from an intentionally choreographed football space can have a significant impact upon his life. It is clear from our participants' responses that the removal of the disciplinary techniques common to football can be disorienting for a player when he retires. As a result, retirees can face an initially challenging period because of their previous reliance upon the powerful sustaining effects of the disciplinary techniques prevalent in the football context. Without the constant prescription of activities or the regular reinforcement of expected behaviours (such as those mentioned by our participants above), retired players are often at a loss as to how to establish direction in their new roles within society - especially at the outset of their retirement.

Foucault's instruments of discipline, his 'Means of correct training', can also help to highlight the disciplinary nature of a footballer's career and provide insight into why retirement can be challenging. For example, when discussing 'hierarchical observation', Foucault explained that in workshops and factories, constant supervision 
instils discipline by taking into account "the activity of men, their skill, the way they set about their tasks, their promptness, their zeal, their behaviour” (Foucault, 1991: 174).

We also suggest that the removal of the continuous supervision found in working football significantly contributes to a retiring player's disorientation. Foucault (1991) observed that because discipline coerces by observation, certain architectural arrangements (for example the lines and boundaries of a football practice field or the position of a manager's office within a training facility) can have powerful coercive effects upon resident bodies. This quote from Harry illustrates how he felt while performing his function as a working player under the conditions of constant supervision instilled at his football club,

The coaches always had an eye on you. What time you turned up - when you left. During training and matches they were always up on the side or in the dugout, watching, and whispering to each other - so, every time you made a bad touch - you thought - shit. And, you were always shit scared they would spot if you didn’t track your runner. Always being watched we were. I have to say although you got used to it, the fact that you are always being watched never really went away.

As a highly skilled manual labourer (Roderick, 2006), the continuous supervision that Harry described was enforced by coaches and managers as 'specialized personnel' (Foucault, 1991: 174). Hierarchical observation is therefore enacted through a 
combination of the coaches' and managers' position of expertise and their physical positioning within the architectural spaces of the working football environment. This strategic arrangement allows 'no zone of shade and constantly supervises' (176) and has a powerful, but importantly, the constant and reliable effect of discipline upon the supervised resident bodies.

Foucault's second instrument of discipline was what he called 'normalising judgement'. He saw that another way that discipline could be worked onto the individual body was through exposure to repeated strategies of normalisation. By ensuring that even the slightest departures from correct behaviour are subject to punishment, or by ensuring that expected behaviours and outputs are repeatedly rewarded, discipline is constantly exercised. Continuous supervision (enabled through hierarchical observation) is utilised to record deviance and reward conformity, consequently normalising the behaviour of the individual. For a footballer the constant supervision and comparison to normalised and expected behaviours had significant consequences. Those who observe a footballer establish a 'penal accountancy' of his football related behaviours - an invisible 'punitive balance sheet' composed of all manner of actions. Everything he performed, from miles ran, tackles missed/won, fouls conceded, goals scored, reactions to criticism from coaching staff/team mates, would have been observed and considered. To ensure his conformity, but also to ensure he maintained access to the rewards associated with this conformity, a footballer would 
have accepted being fined for arriving late, would have displayed normalised, hypercompetitive behaviour, and would have obediently engaged in any prescribed training activity by his superiors. As a result of this constant surveillance, the punishment associated with it, as well as the regular gratification for displaying normalised and expected behaviours, disciplinary power was heavily integrated into a footballer's everyday role. The consequence of this was that it normalised his body and his behaviour, and produced him as a docile footballer.

Foucault's (1991: 184) final instrument of discipline was the combination of hierarchical observation and normalising judgement - ‘the examination'. An examination is a highly ritualised event that "makes it possible to qualify, to classify and to punish”. The examination makes it possible to extract and constitute knowledge surrounding an individual - in this case - a footballer. This quote from George highlights just how many examinations a footballer’s day to day life includes. I haven’t been for many job interviews, but when I was a footballer it felt like I was like, going for one every day you know? Right from when you were a kid on trial - then when you're at a club, the smart clothes you had to wear for home games - to the tight time keeping all over the place - like if you had a meeting with the gaffer...Other things used to irritate me like them keeping a tab on your height and weight at the start and end of pre-season. Then them springing fitness testing on us throughout the year, bleep tests and all that. I could keep going - a 
medical before a new contract, all that stuff, it was constant.

Clearly a working footballer is examined on a regular basis. Foucault (1991: 189) observed that "the examination that places the individual in a field of surveillance also situates them in a network of writing; it engages them in a whole mass of documents that capture and fix them”. The footballer who is regularly examined has established for him a 'knowledge' of his capabilities as a player. This knowledge, produced via multiple examinations, becomes intimately connected to the player's identity, and can therefore be used by the coach to further discipline, punish, and sustain a working player.

Another quote from Lawrence shows that retirement was a significant challenge as it left him without a clear understanding of the future,

It left me stuck in a rut. I thought....what do I do with the rest of my days? Literally what am I going to do? There was quite a big phase where I had no focus. Not through want, but because I had nothing to focus on. I had no-one telling me what I needed to do next.

For us Lawrence's quote is revealing as it highlights that in his post-football life, a retired player conducts his day to day existence in a space no longer occupied or constantly observed by the once familiar arrangement of managers, coaches, teammates or club staff. He no longer has his activities or behaviour regularly examined, and as a result, he is no longer repeatedly exposed to football's disciplinary practices or it's 
'Means of Correct Training'. We suggest that the removal of disciplinary techniques and instruments associated with retirement is central to the emotional challenge a retiring player endures. The removal of hierarchical observation and normalising judgement (both punishment and gratification) and their combination in the examination -(experienced on a regular basis as a footballer), significantly contributes to a player’s disorientation upon his retirement. The "innumerable mechanisms” of surveillance found within his fixed football club that had formed around him "an apparatus of observation, recording, and training” (Foucault, 1991: 173) have, in his retirement, been removed. We argue that for Lawrence, much like Michael, retirement (initially at least) caused significant confusion, because, without the imposed daily surveillance associated with the football space, and the training he experienced within it, he began to feel his life changing and becoming increasingly unstable, or 'without focus'. We suggest that the reduction in surveillance for footballers, coupled with the aforementioned withdrawal of disciplinary techniques experienced by Brian and Liam, can lead to increased uncertainty in the retired footballer's life. Accordingly, we believe that the effects of disciplinary practices and instruments of surveillance should be prioritised when attempting to understand athletes' retirement experiences.

We feel that it is important we are clear that the players interviewed insisted that these feelings of challenge had not occurred in isolation. And, that despite these numerous recollections of 'challenge', the participants were often simultaneously filled 
with a sense of distinct 'relief' upon their retirement. In the next section we explore these feelings of relief.

\section{Relief}

So far, we have explained that challenging times were brought on by the sudden loss of structure, organization and control that our participants had become so reliant upon during their exposure to football’s overarching disciplinary apparatus. In this section we suggest that at the same time there were strong sensations of relief experienced by our participants that was born out of the relinquishment of pressure, expectation and the level of sacrifice that the overtly disciplinary arrangement of working football attempts to preserve in its professionals. More specifically, we suggest that a sensation of relief occurs as a result of the overt and punitive discipline associated with the role of the footballer being removed. Therefore in what follows, similar to how we showed that the challenges related to retiring from football can be associated with players’ removal from football’s disciplinary apparatus, we show how players’ sensations of relief related to retiring from football can also be associated with their removal from football's disciplinary apparatus. The two quotes below from Eli and Dean reveal the relief experienced at no longer having to perform as a professional footballer:

Interviewer: What is your relationship with football like now? 
Eli: Nothing. Don’t play it. Not kicked a ball. I don’t miss it. I don’t miss people telling me what they think of me, that I wasn’t very good. My Dad always questions me 'Don’t you miss it?' and I say 'I've had enough of it'. It's a fact. I don't want to be associated with people who constantly judge you.

Interviewer: Do you prefer the job you have now?

Eli: Yeah, because I hated the scrutiny. I do not miss the fans and the press slating me, or the manager hammering me. I'm done with that.

Similarly Dean's account of his first job away from football also highlights a sense of relief:

I started working in a warehouse and the difference...I felt brilliant for six months. It was the best feeling I've ever had in a job, ever. Because, I'd gone from football, being under pressure every day I got u:Going from training, knowing I had to perform, to working where I thought 'I do not give a fuck...I haven’t got to do anything...I'm just going to chuck a few boxes on'. No-one was there telling me I was chucking the boxes on in the wrong way, and it was just great to have that.

Both Eli and Dean have explained that no longer having to experience the surveillance typical to a football setting came as a great relief. No longer being placed under the constant and normalizing 'scrutiny' and 'judgment' from the fixed hierarchy of fans, media and superiors that surrounded his club has allowed Eli to abandon football 
completely. Foucault (1991: 176) identified that “although surveillance rests upon the individual, its functioning is that of a network of relations from the top to the bottom”. We believe that this is an accurate description of the network that surrounded Eli, and by leaving this coercive network of surveillance, he was released from a constraining disciplinary arrangement. Dean also explained how he constantly felt pressure in his previous football environment. This is unsurprising as Foucault (1991) noted that even in an intensely disciplinary context the slightest departures from the correct or normalized behaviours are subject to punishment. For example during his career, any mistakes Dean made would have led to him being subjected to abuse from the coaching staff, or even facing a loss of his position (risking becoming a 'reject', Cushion and Jones, 2006). The 'normalizing judgment' common to a football space operates as a corrective instrument of discipline. As mentioned, the constant presence of this corrective mechanism meant that any offence committed was a failure to conform, which in turn, led to punishment. Eli and Dean's sense of relief in retirement must therefore come as no surprise. We believe that these men are reveling in their release from what Foucault (1991: 183) identified as the "perpetual penality that traverses all points and is supervised at every instant”. In his new role, Dean is no longer subjected to any punitive consequences as a result of a deviation from 'chucking boxes on' correctly. This is in direct comparison to his systematic experience of the punitive consequences associated with 'the examinations' that are regularly experienced during a 
football career (e.g., the fitness testing, medical testing, training, matches that George mentioned in the previous section). While it is true that working in a warehouse is a classic example of a disciplinary space, for Dean, following his immediate removal from football, it was a space where the impact of disciplinary power, due to the comparatively weak instruments of surveillance, was not so keenly felt.

Many of the retirees also mentioned how difficult they found it during their careers to escape the constant, competitive nature of their football lives. Michael highlighted the constraining relational nature that typifies the 'constant' feeling of the culture footballers occupy.

It doesn't stop. In the car - on the way to training, there’s banter. In the changing room you're always giving it out or taking it. During training you're looking over your shoulder making sure you do things right- then there's banter about what happened during training in the showers ...Knock off early for a round of golf with the lads - guess what - more competition and banter about football. Get home for tea - text messages from the players taking the piss about whoever. A night out? More of the same. Lying in bed thinking about niggling injuries and contracts. It’s endless - and sometimes you just wanted something else.

The constant imposition of self-regulatory discipline and the 'omni-disciplinary' presence of team mates and coaching staff means that the 'twenty-four seven football culture', defined by unceasing competition (Giulianotti, 1999) and the need to further 
one’s 'rank', ensured that players 'couldn’t relax'. This round the clock one-upmanship, coupled with the constant day-to-day supervision from specialised personnel, (discussed by Harry above), generated the feeling of being unable to escape the 'football bubble'. As a result, and to no real surprise, retirees expressed relief at being able to develop new relationships and to interact with a broader spectrum of people after leaving football. For example, Andrew explained that,

I made a conscious decision to get myself out of the football bubble...meeting new people who weren’t in football was amazing for me. It got me away from the twenty-four-seven football culture, where you live with the lads and see them all the time. I used to go to all the footballers' houses and it was twentyfour seven banter and you just couldn't relax, you couldn’t have a serious conversation. For the most part it was all about one-upmanship...There was no way you could have a conversation like the one we are having now...so by living with other people, you can interact like real people do and meet new people who are more like minded.

The constant discipline and its 'omni-disciplinary' presence meant that for Andrew, the life he experienced as a working footballer, defined by unceasing competition (Giulianotti, 1999) and the need to further one’s 'rank', ensured that 'you couldn’t relax'. The 'football bubble' that Andrew mentioned is a typical example of Foucault's (1991) panoptic guard tower. As a footballer, Andrew was constantly exposed to an 
unceasing and inescapable gaze. As a result he self-regulated his own behaviour and independently worked to develop his usefulness and compliant behavior. This came at a cost, as Andrew felt excluded from alternative ways of knowing himself or using his body. The player that leaves football is removed from the intense and specific 'panoptic gaze' of a hypercompetitive lifestyle that previously managed to pervade the multiple contexts of his life. As a result of the removal associated with retirement, the techniques and instruments of discipline that previously rendered the player docile begin to wane, and their immediate effect upon the player significantly lessens. Andrew’s quote also demonstrates how existing within this panoptic context retarded the development of the alternative skills required to integrate with the expectations of new social spaces. Consequently, when permitted to 'meet like-minded people' in retirement, he experienced a sense of pleasure and release.

In retirement, the footballer leaves an arrangement of disciplinary power and the punitive effects of this discipline begin to fade over time. As the docility of each player erodes, he is then allowed the time and space to develop alternative skills. He is eventually permitted to recreate his retiring self (Crockett, 2014), in order to perform appropriately in the new places and spaces he finds himself within. However, a retired player is also forced to seek alternative means to sustain his newly fragmented identity as he can no longer rely upon the sustaining characteristics of his previously disciplinary lifestyle. We believe that this conundrum is reflective of the complexity of 
the sports retirement experience. As such, in this article that is why we have attempted to make sense of sport retirement as a dynamic and multifaceted experience and process.

\section{Conclusion}

Existing Foucauldian analyses have established that there are significant and powerful implications for bodies that operate within elite sport due to the regular and normal imposition of a range of disciplinary techniques and instruments (e.g., Shogan, 1999). In this study we have built upon these key texts' findings to examine how 'docile footballing bodies' experience retirement. Our results indicate that there is a strong association between football's culture of coach control and player-conformity and how retirement from football is understood and experienced by players. In this regard, the experiences of challenge and relief in retirement that we have reported should be read as an effect of the initial reduction or removal from the sustaining and punitive characteristics of football's disciplinary framework.

Specifically, throughout this paper we have tried to argue that footballers’ retirement experiences are strongly shaped and influenced by various arrangements of disciplinary power operating within their particular sporting context. As a result we believe we are in a position to suggest some new and innovative strategies, for both practitioners and researchers, that might counter the problematic effects that discipline might have upon the athletic body (retired or otherwise). To begin, Foucauldian logic 
dictates that to disrupt the negative consequences experienced in retirement, it is perhaps football's hierarchy or leaders (managers/coaches/development officers), that is those who are ethically responsible for the development of players, who need to carefully consider how a player experiences his career. For example, coaches could be made more aware of the docile making effects that many of their everyday practices have on their players’ bodies and as a result begin to coach differently (Denison, Mills, \& Konoval, 2015).

We recognise that to ask a football coach to begin to 'Coach with Foucault' and to abandon the many accepted and common-sense disciplinary practices that have become so taken-for-granted across all football cultures is no easy task. It is a challenging and dis-orientating undertaking that will require the coach to undergo a significant personal and social transformation, a transformation that would require a coach to abandon a range of controlling and aggressive coaching practices (Cushion and Jones, 2006) and focus instead on developing less disciplinary coaching practices. If a reduction of the making of 'docile footballing bodies' is to ever occur, if there is ever to be real and lasting change for current and former players, then the development and implementation of new ways of coaching must become a priority for all who are involved in the making of football bodies, in particular sport scientists and coach developers. Furthermore, our findings also suggest that the assumption that elite sport automatically builds character requires further attention. This is because elite 
performance sport is, in reality, far removed from 'real life'. Accepting the taken-forgranted notion that coaches automatically prepare athletes for 'real life' through the application of 'appropriate’ disciplinary strategies, is, we suggest, very dangerous. And, as we have highlighted in this paper, is an assumption that justifies the production of athletes through problematic disciplinary practices - an arrangement that we have identified as having lasting consequences for the athlete.

To conclude, Foucault’s (1991) disciplinary analysis has allowed us to interrogate the dominant practices and relationships that compose working football. This interrogation has revealed significant links between the sustaining and punitive effects of discipline present in football and how retirement from football is experienced. We therefore emphasise the importance of coaches developing an appreciation of the many unseen effects that disciplinary power can have upon footballers' bodies both during and after their careers. This necessarily will require the continued re-imagination of football's deeply entrenched and oftentimes problematic coaching practices and relationships. 


\section{References}

Avner Z, Jones L and Denison J (2014) Poststructuralism. In: Nelson L, Groom R and Potrac P (eds) Research Methods in Sports Coaching. London: Routledge, p:42-51.

Barker-Ruchti N and Tinning R (2010) Foucault in leotards: Corporeal discipline in women's artistic gymnastics. Sociology of Sport Journal 27: 229-250.

Blinde E and Stratta T (1992) The sport career death of college athletes: Involuntary and unanticipated sport exits. Journal of Sport Behaviour 15: 3-20.

Brown G and Potrac P (2009) 'You've not made the grade son': de-selection and identity disruption in elite level football. Soccer and Society 10: 143-159.

Christensen M (2009) “An eye for Talent”: Talent identification and the "practical sense” of top level soccer coaches. Sociology of Sport Journal 26: 365-382.

Coakley J (1983) Leaving competitive sport: Retirement or rebirth? Quest 35: 1-11.

Crockett H (2014) I had no desire to be having this battle with this faceless man on the soccer field anymore: exploring the ethics of sport retirement. Sociology of Sport Journal 31 (2): 185-201.

Curran C (2015) Post-playing careers of Irish-born footballers in England, 1945-2010. Sport in Society doi:10.1080/17430437.2015.1042970cc

Cushion C and Jones R (2006) Power, discourse, and symbolic violence in professional youth soccer: The case of Albion football club. Sociology of Sport Journal 23:142-161.

Denison J (2007) Social theory for coaches: A Foucauldian reading of one athlete's poor performance. International Journal of Sports Science and Coaching 2: 369-383.

Denison J and Avner Z (2011) Positive coaching: Ethical practices for athlete development. Quest, 63: 209-227.

Denison J, Mills J and Konoval T (2015) Sports’ disciplinary legacy and the challenge of 'coaching differently'. Sport Education and Society doi:10.1080/13573322.2015.1061986. 
Foucault M (1991) Discipline and punish: The birth of a prison. London: Penguin Books.

Fuller R (2014) Transition Experiences out of Intercollegiate Athletics: A MetaSynthesis. The Qualitative Report 19(46): 1-15.

Gearing B (1997) More than a game: The experience of being a professional footballer in Britain. Oral History 25: 63-70.

Giulianotti R (1999) Football: A Sociology of the Global Game. London: Polity Press.

Gordan S and Lavallee D (2004) Career transitions in competitive sport. In:Will T and Summers J (eds) Sport Psychology: Theory, Applications and Issues (2nd ed).

Milton:Wiley, p:584-610.

Heikkala J (1993) Discipline and excel: Technologies of self and body and the logic of competing. Sociology of Sport Journal 10: 397-412.

Holstein J and Gubrium J (1995) The Active Interview. Qualitative research methods series (Vol. 37). Thousand Oaks: Sage Publications.

Johns D and Johns J (2000) Surveillance, subjectivism and technologies of power: An analysis of the discursive practice of high performance sport. International Review for the Sociology of Sport 35: 219-234.

Markula P and Pringle R (2006) Foucault, Sport, and Exercise: Power, Knowledge, and Transforming the Self. London: Routledge.

Markula P and Silk M (2011) Qualitative Research for Physical Culture. New York: Palgrave MacMillan.

McGillivray D, Fearn R and McIntosh A (2005) Caught up in the beautiful game: A case study of Scottish professional footballers. Journal of Sport and Social Issues 29: 102-123.

Mitchell T, Nesti M, Richardson D, Midgley A, Eubank M, and Littlewood M (2014) Exploring athletic identity in elite-level English youth football: a cross section. Journal of Sports Sciences 32 (13): 1294-1299.

Patton M (2002) Qualitative research and evaluation methods (3rd ed). London: Sage Publications. 
Roderick M (2006) The Work of Professional Football: A Labour of Love? London: Routledge.

Roderick M (2012) An unpaid labour of love: Professional footballers, family life, and the problem of job relocation. Journal of Sport and Social Issues 36: 317-338.

Shogan D (1999) The Making of High Performance Athletes: Discipline, Diversity, and Ethics. Canada: University of Toronto Press.

Stier J (2007) Game, name and fame - afterwards will I still be the same? A social psychological study of career, role exit and identity. International Review for the Sociology of Sport 42: 99-111.

Turner A, Barlow J and Heathcote-Elliot C (2000) Long-term health impact of playing professional football in the UK. British Journal of Sports Medicine 34: 332-336. 